\title{
Allogeneic Adenovirus-specific Cytotoxic T Lymphocytes
}

National Cancer Institute

\section{Source}

National Cancer Institute. Allogeneic Adenovirus-specific Cytotoxic T Lymphocytes. NCI

Thesaurus. Code C101796.

A population of allogeneic cytotoxic T-lymphocytes (CT Ls) specifically reactive to human adenovirus (Ad) with potential immunomodulating and anti-adenoviral activities. Upon immunoprophylactic adoptive cell therapy, infusion of allogeneic Ad-specific CT Ls may help reconstitute $\mathrm{Ad}$-specific $\mathrm{CT} L$ responses in patients at risk of developing Ad infections after allog eneic stem cell transplant or in Ad-infected immunocompromised hosts. These allogeneic Ad-specific CT Ls are prepared by multiple rounds of stimulation with donor peripheral blood mononuclear cells and lymphoblastoid cell lines that have been transduced with Ad5f35, a recombinant adenoviral vector carrying no transgene. 